\title{
A physical, genetic and functional sequence assembly of the barley genome
}

The International Barley Genome Sequencing Consortium*

Barley (Hordeum vulgare L.) is among the world's earliest domesticated and most important crop plants. It is diploid with a large haploid genome of 5.1 gigabases (Gb). Here we present an integrated and ordered physical, genetic and functional sequence resource that describes the barley gene-space in a structured whole-genome context. We developed a physical map of $4.98 \mathrm{~Gb}$, with more than $3.90 \mathrm{~Gb}$ anchored to a high-resolution genetic map. Projecting a deep whole-genome shotgun assembly, complementary DNA and deep RNA sequence data onto this framework supports 79,379 transcript clusters, including 26,159 'high-confidence' genes with homology support from other plant genomes. Abundant alternative splicing, premature termination codons and novel transcriptionally active regions suggest that post-transcriptional processing forms an important regulatory layer. Survey sequences from diverse accessions reveal a landscape of extensive single-nucleotide variation. Our data provide a platform for both genome-assisted research and enabling contemporary crop improvement.

Cultivated barley, derived from its wild progenitor Hordeum vulgare ssp. spontaneum, is among the world's earliest domesticated crop species ${ }^{1}$ and today represents the fourth most abundant cereal in both area and tonnage harvested (http://faostat.fao.org). Approximately three-quarters of global production is used for animal feed, $20 \%$ is malted for use in alcoholic and non-alcoholic beverages, and $5 \%$ as an ingredient in a range of food products ${ }^{2}$. Barley is widely adapted to diverse environmental conditions and is more stress tolerant than its close relative wheat ${ }^{3}$. As a result, barley remains a major food source in poorer countries ${ }^{4}$, maintaining harvestable yields in harsh and marginal environments. In more developed societies it has recently been classified as a true functional food. Barley grain is particularly high in soluble dietary fibre, which significantly reduces the risk of serious human diseases including type II diabetes, cardiovascular disease and colorectal cancers that afflict hundreds of millions of people worldwide ${ }^{5}$. The USA Food and Drug Administration permit a human health claim for cellwall polysaccharides from barley grain.

As a diploid, inbreeding, temperate crop, barley has traditionally been considered a model for plant genetic research. Large collections of germplasm containing geographically diverse elite varieties, landraces and wild accessions are readily available ${ }^{6}$ and undoubtedly contain alleles that could ameliorate the effect of climate change and further enhance dietary fibre in the grain. Enriching its broad natural diversity, extensive characterized mutant collections containing all of the morphological and developmental variation observed in the species have been generated, characterized and meticulously maintained. The major impediment to the exploitation of these resources in fundamental and breeding science has been the absence of a reference genome sequence, or an appropriate enabling alternative. Providing either of these has been the primary research challenge to the global barley community.

In response to this challenge, we present a novel model for delivering the genome resources needed to reinforce the position of barley as a model for the Triticeae, the tribe that includes bread and durum wheats, barley and rye. We introduce the barley genome gene space, which we define as an integrated, multi-layered informational resource that provides access to the majority of barley genes in a highly structured physical and genetic framework. In association with comparative sequence and transcriptome data, the gene space provides a new molecular and cellular insight into the biology of the species, providing a platform to advance gene discovery and genome-assisted crop improvement.

\section{A sequence-enriched barley physical map}

We constructed a genome-wide physical map of the barley cultivar (cv.) Morex by high-information-content fingerprinting ${ }^{7}$ and contig assembly $^{8}$ of 571,000 bacterial artificial chromosome (BAC) clones $(\sim 14$-fold haploid genome coverage) originating from six independent BAC libraries ${ }^{9}$. After automated assembly and manual curation, the physical map comprised 9,265 BAC contigs with an estimated N50 contig size of 904 kilobases and a cumulative length of $4.98 \mathrm{~Gb}$ (Methods, Supplementary Note 2). It is represented by a minimum tiling path (MTP) of 67,000 BAC clones. Given a genome size of $5.1 \mathrm{~Gb}^{10}$, more than $95 \%$ of the barley genome is represented in the physical map, comparing favourably to the 1,036 contigs that represent $80 \%$ of the $1 \mathrm{~Gb}$ wheat chromosome $3 \mathrm{~B}^{11}$.

We enhanced the physical map by integrating shotgun sequence information from 5,341 gene-containing ${ }^{12,13}$ and 937 randomly selected BAC clones (Methods, Supplementary Notes 2 and 3, and Supplementary Table 4), and 304,523 BAC-end sequence (BES) pairs (Supplementary Table 3). These provided 1,136 megabases (Mb) of genomic sequence integrated directly into the physical map (Supplementary Tables 3 and 4). This framework facilitated the incorporation of whole-genome shotgun sequence data and integration of the physical and genetic maps. We generated whole-genome shotgun sequence data from genomic DNA of cv. 'Morex' by short-read Illumina GAIIx technology, using a combination of 300 base pairs (bp) paired-end and $2.5 \mathrm{~kb}$ mate-pair libraries, to $>50$-fold haploid genome coverage (Supplementary Note 3.3). De novo assembly resulted in sequence contigs totalling $1.9 \mathrm{~Gb}$. Due to the high proportion of repetitive DNA, a substantial part of the whole-genome shotgun data collapsed into relatively small contigs characterized by exceptionally high read depths. Overall, 376,261 contigs were larger than $1 \mathrm{~kb}$ $(\mathrm{N} 50=264,958$ contigs, N50 length $=1,425 \mathrm{bp})$. Of these, 112,989

*A list of authors and their affiliations appears at the end of the paper. 
(308 Mb) could be anchored directly to the sequence-enriched physical map by sequence homology.

We implemented a hierarchical approach to further anchor the physical and genetic maps (Methods, Supplementary Note 4). A total of 3,241 genetically mapped gene-based single-nucleotide variants (SNV) and 498,165 sequence-tag genetic markers ${ }^{14}$ allowed us to use sequence homology to assign 4,556 sequence-enriched physical map contigs spanning $3.9 \mathrm{~Gb}$ to genetic positions along each barley chromosome. An additional 1,881 contigs were assigned to chromosomal bins by sequence homology to chromosome-arm-specific sequence data sets ${ }^{15}$ (Supplementary Note 4.4). Thus, 6,437 physical map contigs totalling $4.56 \mathrm{~Gb}$ (90\% of the genome), were assigned to chromosome arm bins, the majority in linear order. Non-anchored contigs were typically short and lacked genetically informative sequences required for positional assignment.

Consistent with genome sequences of other grass species ${ }^{16}$ the pericentromeric and centromeric regions of barley chromosomes exhibit significantly reduced recombination frequency, a feature that compromises exploitation of genetic diversity and negatively impacts genetic studies and plant breeding. Approximately $1.9 \mathrm{~Gb}$ or $48 \%$ of the genetically anchored physical map $(3.9 \mathrm{~Gb})$ was assigned to these regions (Fig. 1 and Supplementary Fig. 11).

\section{Repetitive nature of the barley genome}

A characteristic of the barley genome is the abundance of repetitive $\mathrm{DNA}^{17}$. We observed that approximately $84 \%$ of the genome is comprised of mobile elements or other repeat structures (Supplementary

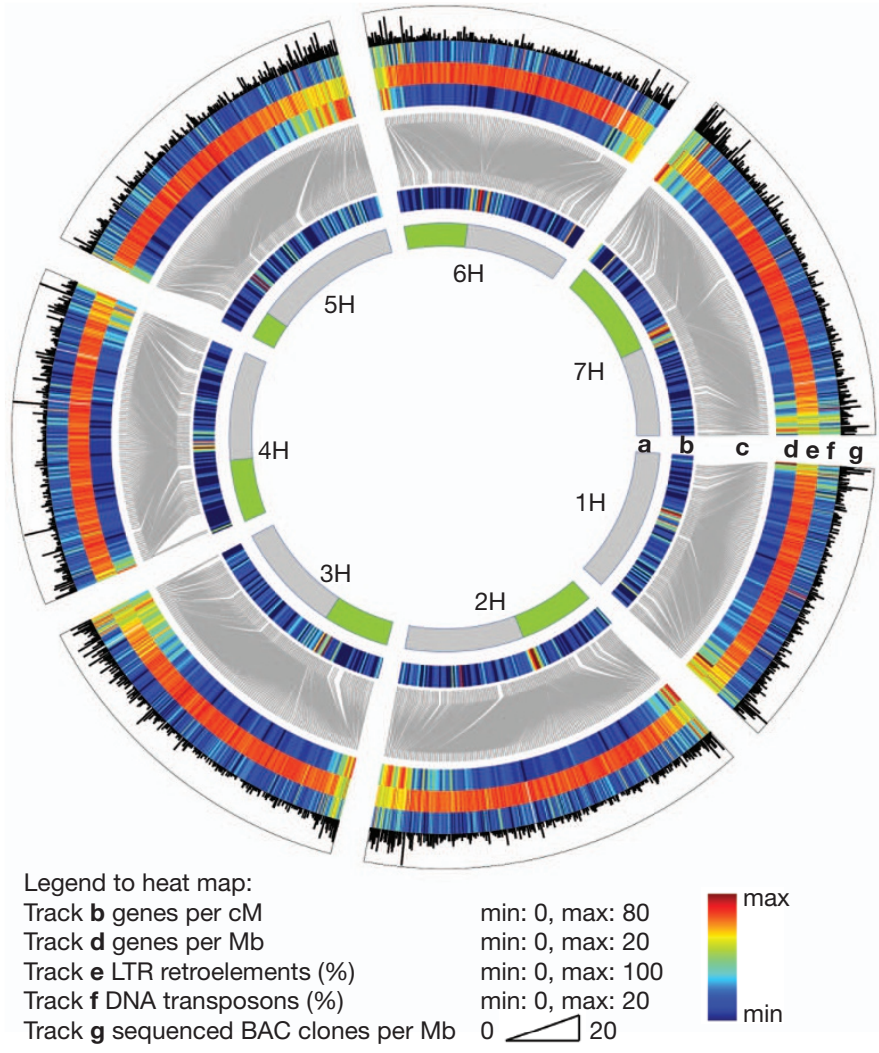

Figure $\mathbf{1} \mid$ Landscape of the barley gene space. Track a gives the seven barley chromosomes. Green/grey colour depicts the agreement of anchored fingerprint (FPC) contigs with their chromosome arm assignment based on chromosome-arm-specific shotgun sequence reads (for further details see Supplementary Note 4). For $1 \mathrm{H}$ only whole-chromosome sequence assignment was available. Track $\mathbf{b}$, distribution of high-confidence genes along the genetic map; track c, connectors relate gene positions between genetic and the integrated physical map given in track $\mathbf{d}$. Position and distribution of track e class I LTR-retroelements and track $\mathbf{f}$ class II DNA transposons are given. Track $\mathbf{g}$, distribution and positioning of sequenced BACs.
Note 5). The majority (76\% in random BACs) of these consists of retrotransposons, $99.6 \%$ of which are long terminal repeat (LTR) retrotransposons. The non-LTR retrotransposons contribute only $0.31 \%$ and the DNA transposons $6.3 \%$ of the random BAC sequence. In the fraction of the genome with a high proportion of repetitive elements, the LTR Gypsy retrotransposon superfamily was 1.5 -fold more abundant than the Copia superfamily, in contrast to observations in both Brachypodium ${ }^{18}$ and rice $^{19}$. However, gene-bearing BACs were slightly depleted of retrotransposons, consistent with Brachypodium $^{18}$ where young Copia retroelements are preferentially found in gene-rich, recombinogenic regions from which inactive Gypsy retroelements have been lost by LTR-LTR recombination. Overall, we see reduced repetitive DNA content within the terminal $10 \%$ of the physical map of each barley chromosome arm (Fig. 1). Class I and II elements show non-quantitative reverse-image distribution along barley chromosomes (Fig. 1), a feature shared with other grass genomes ${ }^{16,20}$ and shown by fluorescence in situ hybridization (FISH) mapping ${ }^{17}$. Not surprisingly, the whole-genome shotgun assembly shows a lower abundance of LTR retrotransposons (average $53 \%)$ than gene-bearing BACs. That LTR retrotransposons are long $(\sim 10 \mathrm{~kb})$, highly repetitive and often nested ${ }^{21}$ supports our assumption that short reads either collapsed or did not assemble. Short interspersed elements (SINEs) ${ }^{22}$, short (80-600 bp) non-autonomous retrotransposons that are highly repeated in barley, showed no differential exclusion from the assemblies. However, miniature invertedrepeat transposable elements (MITEs), small non-autonomous DNA transposons ${ }^{23}$, were twofold enriched in the whole-genome shotgun assemblies compared with BES reads or random BACs, consistent with the gene richness of the assemblies and their association with genes $^{23}$. Both MITEs and SINEs are 1.5 to 2-fold enriched in genebearing BACs which could indicate that SINEs are also preferentially integrated into gene-rich regions, or because they are older than LTR retroelements, may simply remain visible in and around genes where retro insertions have been selected against.

\section{Transcribed portion of the barley genome}

The transcribed complement of the barley gene space was annotated by mapping 1.67 billion RNA-seq reads $(167 \mathrm{~Gb})$ obtained from eight stages of barley development as well as 28,592 barley full-length $\mathrm{cDNAs}^{24}$ to the whole-genome shotgun assembly (Methods, Supplementary Notes 6, 7 and Supplementary Tables 20-22). Exon detection and consensus gene modelling revealed 79,379 transcript clusters, of which 75,258 (95\%) were anchored to the whole-genome shotgun assembly (Supplementary Notes 7.1.1 and 7.1.2). Based on a genefamily-directed comparison with the genomes of Sorghum, rice, Brachypodium and Arabidopsis, 26,159 of these transcribed loci fall into clusters and have homology support to at least one reference genome (Supplementary Fig. 16); they were defined as high-confidence genes. Comparison against a data set of metabolic genes in Arabidopsis thalian $a^{25}$ indicated a detection rate of $86 \%$, allowing the barley geneset to be estimated as approximately 30,400 genes. Due to lack of homology and missing support from gene family clustering, 53,220 transcript loci were considered low-confidence (Table 1). Highconfidence and low-confidence barley genes exhibited distinct characteristics: $75 \%$ of the high-confidence genes had a multi-exon structure, compared with only $27 \%$ of low-confidence genes (Table 1 ). The mean size of high-confidence genes was 3,013 bp compared with 972 bp for low-confidence genes. A total of 14,481 low-confidence genes showed distant homology to plant proteins in public databases (Supplementary Notes 7.1.2, 7.1.4 and Supplementary Fig. 18), identifying them as potential gene fragments known to populate Triticeae genomes at high copy number and that often result from transposable element activity ${ }^{26}$.

A total of 15,719 high-confidence genes could be directly associated with the genetically anchored physical map (Supplementary Note 4). An additional 3,743 were integrated by invoking a conservation of synteny model (Supplementary Note 4.5 ) and a further 4,692 by association 
Table 1 | Characteristics of high-confidence and low-confidence gene sets in barley

\begin{tabular}{|c|c|c|}
\hline & High confidence & Low confidence \\
\hline Number of genes & 26,159 & 53,220 \\
\hline Gene loci positioned on barley cultivar & $24,243(93 \%)$ & $51,015(96 \%)$ \\
\hline \multicolumn{3}{|l|}{ Morex assembly* } \\
\hline Single exon & $5,954(25 \%)$ & $37,395(73 \%)$ \\
\hline Multi exon & $18,289(75 \%)$ & $13,620(27 \%)$ \\
\hline Number of distinct exons $\dagger$ & 184,710 & 107,768 \\
\hline Mean number of distinct exons per gene & 7.62 & 2.11 \\
\hline $\begin{array}{l}\text { Number of genes with alternative } \\
\text { transcript variants }\end{array}$ & $13,299(55 \%)$ & $8,214(16 \%)$ \\
\hline umber of predicted transcripts & 62,426 & 69,266 \\
\hline her of transcripts per gene & 2.58 & 1.36 \\
\hline ne locus size (first to last exon) & $3,013 \mathrm{bp}$ & $972 b p$ \\
\hline ript size (UTR, CDS) & $78 \mathrm{bp}$ & $931 \mathrm{bp}$ \\
\hline on size & $454 \mathrm{bp}$ & $536 b p$ \\
\hline $\begin{array}{l}\text { Gene loci not positioned on barley cv. } \\
\text { Morex assembly: }\end{array}$ & $1,916(7 \%)$ & $2,205(4 \%)$ \\
\hline Tagged by unmapped RNA-seq reads & $1,657(86 \%)$ & $1,127(51 \%)$ \\
\hline Not tagged by unmapped RNA-seq reads & $259(14 \%)$ & $1,078(49 \%)$ \\
\hline \multicolumn{3}{|c|}{$\begin{array}{l}\text { * Gene locus representatives are (1) RNA-seq based transcript or (2) barley fl-cDNA that were mappec } \\
\text { to the barley cultivar Morex assembly or tagged by RNA-seq based transcript during clustering. } \\
\dagger \text { Exons of two or more transcripts were counted once if they have identical start and stop positions. } \\
\text { \# Gene locus representatives are barley fl-cDNAs that were not mapped to the barley cultivar Morex } \\
\text { assembly and not matched by any RNA-seq based transcript } \\
\text { CDS, coding sequence. }\end{array}$} \\
\hline
\end{tabular}

with chromosome arm whole-genome shotgun data (Supplementary Note 4.4 and Supplementary Table 15). Importantly, the N50 length of whole-genome shotgun sequence contigs containing high-confidence genes was $8,172 \mathrm{bp}$, which is generally sufficient to include the entire coding sequence, and $5^{\prime}$ and $3^{\prime}$ untranslated regions (UTRs). Overall 24,154 high-confidence genes $(92.3 \%)$ were associated and positioned in the physical/genetic scaffold, representing a gene density of five genes per $\mathrm{Mb}$. Proximal and distal ends of chromosomes are more gene-rich, on average containing 13 genes per $\mathrm{Mb}$ (Fig. 1).

In comparison with sequenced model plant genomes, gene family analysis (Supplementary Note 7.1.3) revealed some gene families that exhibited barley-specific expansion. We defined the functions of members of these families using gene ontology (GO) and PFAM protein motifs (Supplementary Table 25). Gene families with highly overrepresented GO/PFAM terms included genes encoding $(1,3)-\beta$ glucan synthases, protease inhibitors, sugar-binding proteins and sugar transporters. NB-ARC (a nucleotide-binding adaptor shared by APAF- 1 , certain R gene products and CED- $4^{27}$ ) domain proteins, known to be involved in defence responses, were also overrepresented, including 191 NBS-LRR type genes. These tended to cluster towards the distal regions of barley chromosomes (Supplementary Fig. 17), including a major group on barley chromosome $1 \mathrm{HS}$, colocalizing with the $M L A$ powdery mildew resistance gene cluster ${ }^{28}$. Biased allocation to recombination-rich regions provides the genomic environment for generating sequence diversity required to cope with dynamic pathogen populations $s^{29,30}$. It is noteworthy that the highly over-represented $(1,3)$ - $\beta$-glucan synthase genes have also been implicated in plant-pathogen interactions ${ }^{31}$.

\section{Regulation of gene expression}

Deep RNA sequence data (RNA-seq) provided insights into the spatial and temporal regulation of gene expression (Supplementary Note 7.2). We found $72-84 \%$ of high-confidence genes to be expressed in all spatiotemporal RNA-seq samples (Fig. 2a), slightly lower than reported for rice ${ }^{32}$ where $\sim 95 \%$ of transcripts were found in more than one developmental or tissue sample. More importantly, 36-55\% of highconfidence barley genes seemed to be differentially regulated between samples (Fig. 2b), highlighting the inherent dynamics of barley gene expression.

Two notable features support the importance of post-transcriptional processing as a central regulatory layer (Supplementary Notes 7.3 and 7.4). First, we observed evidence for extensive alternative splicing. Of the intron-containing high-confidence barley genes, $73 \%$ had evidence of alternative splicing (55\% of the entire high-confidence set). The spatial and temporal distribution of alternative splicing transcripts deviated significantly from the general occurrence of transcripts in the different tissues analysed (Fig. 2c). Only $17 \%$ of alternative splicing transcripts were shared among all samples, and $17-27 \%$ of the alternative splicing transcripts were detected only in individual samples, indicating pronounced alternative splicing regulation. We found 2,466 premature termination codon-containing $(\mathrm{PTC}+)$ alternative splicing transcripts (9.4\% of high-confidence genes) (Fig. $2 \mathrm{~d}$ and Table 2), similar to the percentage of nonsense-mediated decay (NMD)-controlled genes in a wide range of species ${ }^{33,34}$. Premature termination codons activate the NMD pathway ${ }^{35}$, which leads to rapid degradation of PTC + transcripts, and have been associated with transcriptional regulation during disease and stress response in human and Arabidopsis, respectively ${ }^{34,36-39}$. The distribution of PTC + transcripts was strikingly dissimilar, both spatially and temporally, with only 7.4\% shared and between $31 \%$ and $40 \%$ exclusively observed in only a single sample (Fig. 2d). Genes encoding PTC+-containing transcripts show a broad spectrum of GO terms and PFAM domains and are more prevalent in expanded gene families. These observations support a central role for alternative splicing/NMD-dependent decay of PTC+ transcripts as a mechanism that controls the expression of many different barley genes.

Second, recent reports have highlighted the abundance of novel transcriptionally active regions in rice that lack homology to proteincoding genes or open reading frames $(\mathrm{ORFs})^{40}$. In barley as many as 27,009 preferentially single-exon low-confidence genes can be classified as putative novel transcriptionally active regions (Supplementary Note 7.1.4). We investigated their potential significance by comparing the homology of barley novel transcriptionally active regions with the rice and Brachypodium genomes that respectively represent 50 and 30 million years of evolutionary divergence ${ }^{18}$. A total of 4,830 and 2,450 novel transcriptionally active regions yielded a homology match to the Brachypodium and rice genomes, respectively (intersection of 2,046; BLAST $P$ value $\leq 10^{-5}$ ), indicating a putative functional role in premRNA processing or other RNA regulatory processes ${ }^{41,42}$.

\section{Natural diversity}

Barley was domesticated approximately 10,000 years ago $^{1}$. Extensive genotypic analysis of diverse germplasm has revealed that restricted outcrossing $(0-1.8 \%)^{43}$, combined with low recombination in pericentromeric regions, has resulted in modern germplasm that shows limited regional haplotype diversity ${ }^{44}$. We investigated the frequency and distribution of genome diversity by survey sequencing four diverse barley cultivars ('Bowman', 'Barke', 'Igri' and 'Haruna Nijo') and an H. spontaneum accession (Methods and Supplementary Note 8) to a depth of 5-25-fold coverage, and mapping sequence reads against the barley cultivar 'Morex' gene space. We identified more than 15 million non-redundant single-nucleotide variants (SNVs). H. spontaneum contributed almost twofold more SNV than each of the cultivars (Supplementary Table 28). Up to 6 million SNV per accession could be assigned to chromosome arms, including up to 350,000 associated with exons (Supplementary Table 29). Approximately $50 \%$ of the exonlocated SNV were integrated into the genetic/physical framework (Fig. 3, Supplementary Table 30 and Supplementary Fig. 31), providing a platform to establish true genome-wide marker technology for highresolution genetics and genome-assisted breeding.

We observed a decrease in SNV frequency towards the centromeric and peri-centromeric regions of all barley chromosomes, a pattern that seemed more pronounced in the barley cultivars. This trend was supported by SNV identified in RNA-seq data from six additional cultivars mapped onto the Morex genomic assembly (Supplementary Note 8.2). We attribute this pattern of eroded genetic diversity to low recombination in the pericentromeric regions, which reduces effective population size and consequently haplotype diversity. Whereas 
a

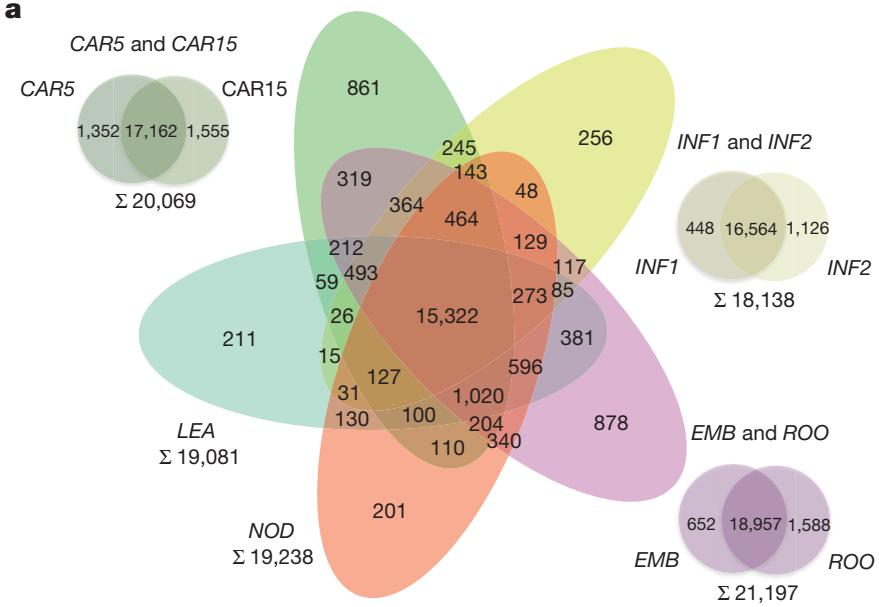

C

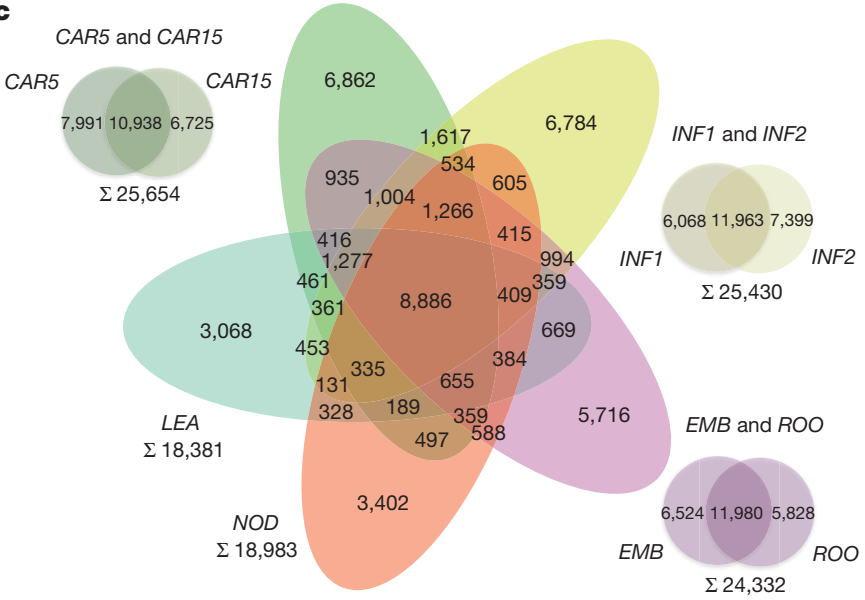

Figure $2 \mid$ Atlas of barley gene expression. a, Barley gene expression in different spatial and temporal RNA-seq samples (Supplementary Notes 6, 7). Numbers refer to high-confidence genes. $\mathbf{b}$, Dendrogram depicting relatedness of samples and colour-coded matrix showing number of significantly upregulated high-confidence genes in pairwise comparisons. $\Sigma$, total number of non-redundant high-confidence genes upregulated in comparison to all other

Table 2 | Alternative splicing and transcripts containing PTCs in high-confidence genes

General statistics of alternative splicing in high-confidence genes

High-confidence genes with RNA-seq data to monitor

24,243

alternative splicing

Predicted transcripts at high-confidence genes

Transcripts with complete CDS structures*

Transcripts with partial CDS structures $\dagger$

Genes with alternative transcripts

Predicted transcripts derived from genes with

alternative splicing

Premature stop codon analysis

Predicted transcripts used for PTC analysis:

Transcripts without PTC

Transcripts containing PTC

PTC caused by intron retention

PTC + transcripts predicted to be NMD****-sensitive

Gene loci incorporating PTC+/NMD transcripts

* Entire predicted coding sequence $(100 \%)$ was transferred to transcript model on barley cultivar Morex contigs.

$\dagger$ Predicted coding sequence could not be completely projected to genomic transcript model (partial mapping of fl-cDNA).

\$ Only transcripts with structures for entire coding sequence on barley cultivar Morex WGS assembly were considered.

$\mathrm{CDS}$, coding sequence.
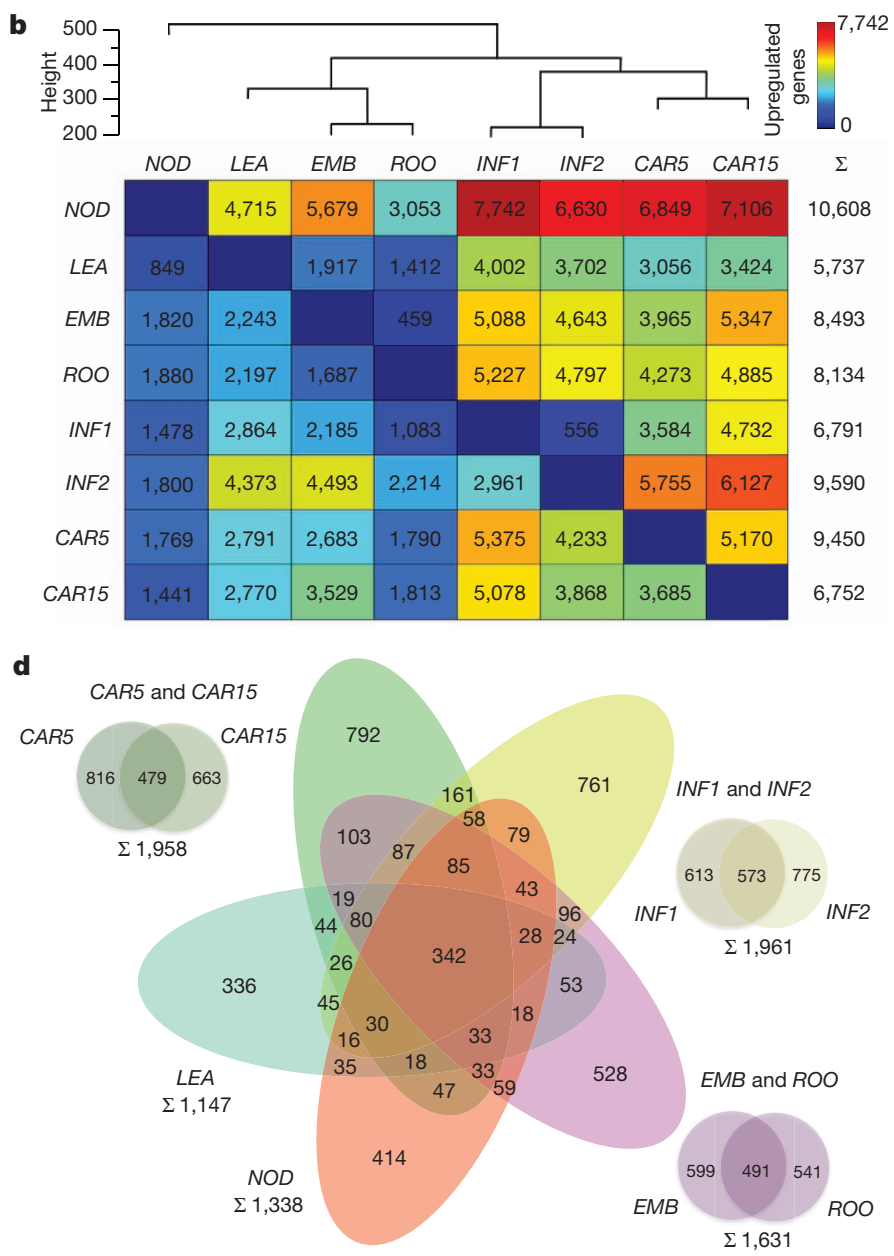

samples. Height, complete linkage cluster distance $\left(\log _{2}\right.$ (fragments per kilobase of exon per million fragments mapped)); see Supplementary Note 7.2.5.1.

c, Distribution and overlap of alternatively spliced barley transcripts between RNA-seq samples. d, Distribution and overlap of alternative splicing transcripts fulfilling criteria for PTC + as detected in different spatial and temporal RNAseq samples (Supplementary Note 7.4).

H. spontaneum may serve here as a reservoir of genetic diversity, using this diversity may itself be compromised by restricted recombination and the consequent inability to disrupt tight linkages between desirable and deleterious alleles. Surprisingly, the short arm of chromosome $4 \mathrm{H}$ had a significantly lower SNV frequency than all other barley chromosomes (Supplementary Fig. 33). This may be a consequence of a further reduction in recombination frequency on this chromosome, which is genetically (but not physically) shortest. Reduced SNV diversity was also observed in regions we interpret to be either the consequences of recent breeding history or could indicate landmarks of domestication (Fig. 3).

\section{Discussion}

The size of Triticeae cereal genomes, due to their highly repetitive DNA composition, has severely compromised the assembly of wholegenome shotgun sequences and formed a barrier to the generation of high-quality reference genomes. We circumvented these problems by integrating complementary and heterogeneous sequence-based genomic and genetic data sets. This involved coupling a deep physical map with high density genetic maps, superimposing deep short-read wholegenome shotgun assemblies, and annotating the resulting linear, albeit punctuated, genomic sequence with deep-coverage RNA-derived data (full-length cDNA and RNA-seq). This allowed us to systematically delineate approximately $4 \mathrm{~Gb}(80 \%)$ of the genome, including more than $90 \%$ of the expressed genes. The resulting genomic framework 


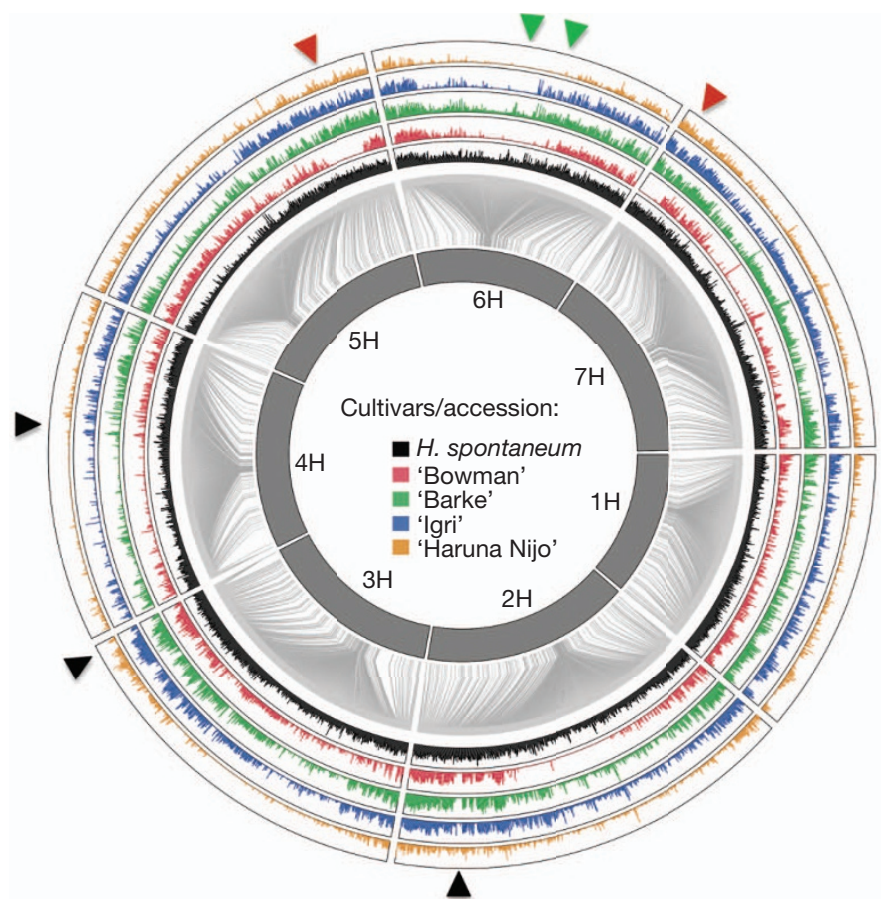

Figure $3 \mid$ Single nucleotide variation (SNV) frequency in barley. Barley chromosomes indicated as inner circle of grey bars. Connector lines give the genetic/physical relationship in the barley genome. SNV frequency distribution displayed as five coloured circular histograms (scale, relative abundance of SNVs within accession; abundance, total number of SNVs in non-overlapping 50 -kb intervals of concatenated 'Morex' genomic scaffold; range, zero to maximum number of SNVs per 50-kb interval). Selected patterns of SNV frequency indicated by coloured arrowheads (for further details see Supplementary Note 8). Colouring of arrowheads refers to cultivar with deviating SNV frequency for the respective region.

provides a detailed insight into the physical distribution of genes and repetitive DNA and how these features relate to genetic characteristics such as recombination frequency, gene expression and patterns of genetic variation.

The centromeric and peri-centromeric regions of barley chromosomes contain a large number of functional genes that are locked into recombinationally 'inert' genomic regions ${ }^{45,46}$. The gene-space distribution highlights that these regions expand to almost $50 \%$ of the physical length of individual chromosomes. Given well-established levels of conserved synteny, this will probably be a general feature of related grass genomes that will have important practical implications. For example, infrequent recombination could function to maintain evolutionarily selected and co-adapted gene complexes. It will certainly restrict the release of the genetic diversity required to decouple advantageous from deleterious alleles, a potential key to improving genetic gain. Understanding these effects will have important consequences for crop improvement. Moreover, for gene discovery, forward genetic strategies based on recombination will not be effective in these regions. Whereas alternative approaches exist for some targets (for example, by coupling resequencing technologies with collections of natural or induced mutant alleles), for most traits it remains a serious impediment. Some promise may lie in manipulating patterns of recombination by either genetic or environmental intervention ${ }^{47}$. Quite strikingly, our data also reveal that a complex layer of post-transcriptional regulation will need to be considered when attempting to link barley genes to functions. Connections between post-transcriptional regulation such as alternative splicing and functional biological consequences remain limited to a few specific examples ${ }^{48}$, but the scale of our observations suggest this list will expand considerably.

In conclusion, the barley gene space reported here provides an essential reference for genetic research and breeding. It represents a hub for trait isolation, understanding and exploiting natural genetic diversity and investigating the unique biology and evolution of one of the world's first domesticated crops.

\section{METHODS SUMMARY}

Methods are available in the online version of the paper.

Full Methods and any associated references are available in the online version of the paper.

Received 2 May; accepted 30 August 2012.

Published online 17 October 2012.

1. Purugganan, M. D. \& Fuller, D. Q. The nature of selection during plant domestication. Nature 457, 843-848 (2009).

2. Blake, T., Blake, V., Bowman, J. \& Abdel-Haleem, H. in Barley: Production, Improvement and Uses (ed. S. E. Ullrich) 522-531 (Wiley-Blackwell, 2011).

3. Nevo, E. etal. Evolution of wild cereals during 28 years of global warming in Israel. Proc. Natl Acad. Sci. USA 109, 3412-3415 (2012).

4. Grando, S. \& Macpherson, H. G. in Proceedings of the International Workshop on Food Barley Improvement, 14-17 January 2002, Hammamet, Tunisia 156 (ICARDA, Aleppo, Syria, 2005).

5. Collins, H. M. et al. Variability in fine structures of noncellulosic cell wall polysaccharides from cereal grains: potential importance in human health and nutrition. Cereal Chem. 87, 272-282 (2010).

6. Bockelman, H. E. \& Valkoun, J. in Barley: Production, Improvement, and Uses (ed. S. E. Ullich) 144-159 (Wiley-Blackwell, 2011).

7. Luo, M.-C. etal. High-throughput fingerprinting of bacterial artificial chromosomes using the snapshot labeling kit and sizing of restriction fragments by capillary electrophoresis. Genomics 82, 378-389 (2003).

8. Soderlund, C., Humphray, S., Dunham, A. \& French, L. Contigs built with fingerprints, markers, and FPC V4.7. Genome Res. 10, 1772-1787 (2000)

9. Schulte, D. et al. BAC library resources for map-based cloning and physical map construction in barley (Hordeum vulgare L.). BMC Genomics 12, 247 (2011).

10. Doležel, J. et al. Plant genome size estimation by flow cytometry: inter-laboratory comparison. Ann. Bot. 82, 17-26 (1998).

11. Paux, E. et al. A physical map of the 1-gigabase bread wheat chromosome $3 \mathrm{~B}$. Science 322, 101-104 (2008)

12. Madishetty, K., Condamine, P., Svensson, J. T., Rodriguez, E. \& Close, T. J. An improved method to identify BAC clones using pooled overgos. Nucleic Acids Res. 35, e5 (2007).

13. Lonardi, S. et al. Barcoding-free BAC pooling enables combinatorial selective sequencing of the barley gene space. preprint at http://arxiv.org/abs/1112.4438 (2011).

14. Poland, J. A., Brown, P. J., Sorrells, M. E. \& Jannink, J.-L. Development of highdensity genetic maps for barley and wheat using a novel two-enzyme genotypingby-sequencing approach. PLOS ONE 7, e32253 (2012).

15. Mayer, K. F. X. et al. Unlocking the barley genome by chromosomal and comparative genomics. Plant Cell 23, 1249-1263 (2011).

16. Schnable, P. S. et al. The B73 maize genome: complexity, diversity, and dynamics. Science 326, 1112-1115 (2009).

17. Wicker, T. et al. A whole-genome snapshot of 454 sequences exposes the composition of the barley genome and provides evidence for parallel evolution of genome size in wheat and barley. Plant J. 59, 712-722 (2009).

18. The International Brachypodium Initiative. Genome sequencing and analysis of the model grass Brachypodium distachyon. Nature 463, 763-768 (2010).

19. International Rice Genome Sequencing Project. The map-based sequence of the rice genome. Nature 436, 793-800 (2005).

20. Paterson, A. H. etal. The Sorghum bicolor genome and the diversification of grasses. Nature 457, 551-556 (2009).

21. Kronmiller, B. A. \& Wise, R. P. TEnest: automated chronological annotation and visualization of nested plant transposable elements. Plant Physiol. 146, 45-59 (2008).

22. Ohshima, K. \& Okada, N. SINEs and LINEs: symbionts of eukaryotic genomes with a common tail. Cytogenet. Genome Res. 110, 475-490 (2005)

23. Wessler, S. R., Bureau, T. \& White, S. LTR-retrotransposons and MITEs: important players in the evolution of plant genomes. Curr. Opin. Genet. Dev. 5, 814-821 (1995).

24. Matsumoto, T.etal. Comprehensive sequence analysis of 24,783 barley full-length cDNAs derived from 12 clone libraries. Plant Physiol. 156, 20-28 (2011).

25. Zhang, P. et al. MetaCyc and AraCyc. Metabolic pathway databases for plant research. Plant Physiol. 138, 27-37 (2005).

26. Wicker, T.etal. Frequent gene movement and pseudogene evolution is common to the large and complex genomes of wheat, barley, and their relatives. Plant Cell 23, 1706-1718 (2011).

27. van der Biezen, E. A. \& Jones, J. D. G. The NB-ARC domain: a novel signalling motif shared by plant resistance gene products and regulators of cell death in animals. Curr. Biol. 8, R226-R228 (1998).

28. Wei, F., Wing, R. A. \& Wise, R. P. Genome dynamics and evolution of the Mla (powdery mildew) resistance locus in barley. Plant Cell 14, 1903-1917 (2002).

29. Halterman, D. A. \& Wise, R.P. A single-amino acid substitution in the sixth leucinerich repeat of barley MLA6 and MLA13 alleviates dependence on RAR 1 for disease resistance signaling. Plant J. 38, 215-226 (2004). 
30. Seeholzer, S. et al. Diversity at the Mla powdery mildew resistance locus from cultivated barley reveals sites of positive selection. Mol. Plant Microbe Interact. $\mathbf{2 3}$ 497-509 (2010).

31. Jacobs, A. K. et al. An Arabidopsis callose synthase, GSL5, is required for wound and papillary callose formation. Plant Cell 15, 2503-2513 (2003).

32. Jiao, Y. et al. A transcriptome atlas of rice cell types uncovers cellular, functional and developmental hierarchies. Nature Genet. 41, 258-263 (2009).

33. Conti, E. \& Izaurralde, E. Nonsense-mediated mRNA decay: molecular insights and mechanistic variations across species. Curr. Opin. Cell Biol. 17, 316-325 (2005)

34. Kalyna, M. et al. Alternative splicing and nonsense-mediated decay modulate expression of important regulatory genes in Arabidopsis. Nucleic Acids Res. 40, 2454-2469 (2012)

35. Lewis, B. P., Green, R. E. \& Brenner, S. E. Evidence for the widespread coupling of alternative splicing and nonsense-mediated mRNA decay in humans. Proc. Natl Acad. Sci. USA 100, 189-192 (2003).

36. Bhuvanagiri, M., Schlitter, A. M., Hentze, M. W. \& Kulozik, A. E. NMD: RNA biology meets human genetic medicine. Biochem. J. 430, 365-377 (2010)

37. Rayson, S. et al. A role for nonsense-mediated mRNA decay in plants: pathogen responses are induced in Arabidopsis thaliana NMD mutants. PLOS ONE 7, e31917 (2012).

38. Riehs-Kearnan, N., Gloggnitzer, J., Dekrout, B., Jonak, C. \& Riha, K. Aberrant growth and lethality of Arabidopsis deficient in nonsense-mediated RNA decay factors is caused by autoimmune-like response. Nucleic Acids Res. 40, 5615-5624 (2012)

39. Jeong, H.-J. et al. Nonsense-mediated mRNA decay factors, UPF1 and UPF3, contribute to plant defense. Plant Cell Physiol. 52, 2147-2156 (2011)

40. Lu, T. et al. Function annotation of the rice transcriptome at single-nucleotide resolution by RNA-seq. Genome Res. 20, 1238-1249 (2010).

41. Guttman, M. \& Rinn, J. L. Modular regulatory principles of large non-coding RNAs. Nature 482, 339-346 (2012)

42. Chinen, M. \& Tani, T. Diverse functions of nuclear non-coding RNAs in eukaryotic gene expression. Front. Biosci. 17, 1402-1417 (2012).

43. Abdel-Ghani, A. H., Parzies, H. K., Omary, A. \& Geiger, H. H. Estimating the outcrossing rate of barley landraces and wild barley populations collected from ecologically different regions of Jordan. Theor. Appl. Genet. 109, 588-595 (2004)

44. Comadran, J. et al. Patterns of polymorphism and linkage disequilibrium in cultivated barley. Theor. Appl. Genet. 122, 523-531 (2011).

45. Close, T. J. et al. Development and implementation of high-throughput SNP genotyping in barley. BMC Genomics 10, 582 (2009).

46. Thiel, T. et al. Evidence and evolutionary analysis of ancient whole-genome duplication in barley predating the divergence from rice. BMC Evol. Biol. 9, 209 (2009).

47. Martinez-Perez, E. \& Moore, G. To check or not to check? The application of meiotic studies to plant breeding. Curr. Opin. Plant Biol. 11, 222-227 (2008)

48. Halterman, D. A., Wei, F. S. \& Wise, R. P. Powdery mildew-induced Mla mRNAs are alternatively spliced and contain multiple upstream open reading frames. Plant Physiol. 131, 558-567 (2003)

Supplementary Information is available in the online version of the paper.

Acknowledgements This work has been supported from the following funding sources: German Ministry of Education and Research (BMBF) grant 0314000 "BARLEX" to K.F.X.M., M.P., U.S. and N.S.; Leibniz Association grant (Pakt f. Forschung und Innovation) to N.S.; European project of the 7th framework programme "TriticeaeGenome" to R.W., A.S., K.F.X.M., M.M. and N.S.; SFB F3705, of the Austrian Wissenschaftsfond (FWF) to K.F.X.M.; ERA-NET PG project "BARCODE" grant to M.M., N.S. and R.W.; Scottish Government/BBSRC grant BB/100663X/1 to R.W., D.M., P.H., J.R., M.C. and P.K.; National Science Foundation grant DBI 0321756 "Coupling EST and Bacterial Artificial Chromosome Resources to Access the Barley Genome" and DBI-1062301 "Barcoding-Free Multiplexing: Leveraging Combinatorial Pooling for High-Throughput Sequencing" to T.J.C. and S.L.; USDA-CSREES-NRI grant 2006-55606-16722 "Barley Coordinated Agricultural Project: Leveraging Genomics, Genetics, and Breeding for Gene Discovery and Barley Improvement" to G.J.M., R.P.W. T.J.C. and S.L.; the Agriculture and Food Research Initiative Plant Genome, Genetics and Breeding Program of USDA-CSREES-NIFA grant 2009-65300-05645 "Advancing the Barley Genome" to T.J.C., S.L. and G.J.M.; BRAIN and NBRP-Japan grants to K.S. Japanese MAFF Grant (TRG1008) to T.M. A full list of acknowledgements is in the Supplementary Information.

Author Contributions See list of consortium authors. R.A., D.S., H.L., B.S., S.T., M.G., F.C. T.N., M.S., M.P., H.G., P.H., T.S., K.F.X.M., R.W. and N.S. contributed equally to their respective work packages and tasks.

Author Information Sequence resources generated or compiled in this study have been deposited at EMBL/ENA or NCBI GenBank. A full list of sequence raw data accession numbers as well as URLs for data download, visualization or search are provided in Supplementary Note 1 and Supplementary Table 1. Reprints and permissions information is available at www.nature.com/reprints. This paper is distributed under the terms of the Creative Commons

Attribution-Non-Commercial-Share Alike license, and the online version of the paper is freely available to all readers. The authors declare no competing financial interests. Readers are welcome to comment on the online version of the paper. Correspondence and requests for materials should be addressed to K.F.X.M (k.mayer@helmholtz-muenchen.de),R.W. (Robbie.Waugh@hutton.ac.uk) or N.S. (stein@ipk-gatersleben.de)

\section{The International Barley Genome Sequencing Consortium (IBSC)}

Principal investigators Klaus F. X. Mayer ${ }^{1}$, Robbie Waugh ${ }^{2}$, Peter Langridge ${ }^{3}$, Timothy $J^{\prime}$ Close $^{4}$, Roger P. Wise ${ }^{5}$, Andreas Graner ${ }^{6}$, Takashi Matsumoto ${ }^{7}$, Kazuhiro Sato ${ }^{8}$, Alan Schulman ${ }^{9}$, Gary J. Muehlbauer ${ }^{10}$, Nils Stein ${ }^{6}$

Physical map construction and direct anchoring Ruvini Ariyadasa ${ }^{6}$, Daniela Schulte ${ }^{6}$ Naser Poursarebani ${ }^{6}$, Ruonan Zhou ${ }^{6}$, Burkhard Steuernagel ${ }^{6}$, Martin Mascher ${ }^{6}$, Uwe Scholz ${ }^{6}$, Bujun Shi ${ }^{3}$, Peter Langridge ${ }^{3}$, Kavitha Madishetty ${ }^{4}$, Jan T. Svensson ${ }^{4}$, Prasanna Bhat ${ }^{4}$, Matthew Moscou ${ }^{4}$, Josh Resnik ${ }^{4}$, Timothy J. Close ${ }^{4}$, Gary J. Muehlbauer ${ }^{10}$, Pete Hedley ${ }^{2}$, Hui Liu ${ }^{2}$, Jenny Morris ${ }^{2}$, Robbie Waugh ${ }^{2}$, Zeev Frenkel $^{11}$, Avraham Korol $^{11}$, Hélène Bergès ${ }^{12}$, Andreas Graner ${ }^{6}$, Nils Stein (leader) ${ }^{6}$

Genomic sequencing and assembly Burkhard Steuernagel ${ }^{6}$, Uwe Scholz ${ }^{6}$, Stefan Taudien $^{13}$, Marius Felder ${ }^{13}$, Marco Groth ${ }^{13}$, Matthias Platzer ${ }^{13}$, Nils Stein (leader) ${ }^{6}$

BAC sequencing and assembly Burkhard Steuernage ${ }^{6}$, Uwe Scholz ${ }^{6}$, Axel Himmelbach ${ }^{6}$, Stefan Taudien ${ }^{13}$, Marius Felder ${ }^{13}$, Matthias Platzer ${ }^{13}$, Stefano Lonardi $^{14}$, Denisa Duma ${ }^{14}$, Matthew Alpert ${ }^{14}$, Francesa Cordero ${ }^{14,22}$, 'Marco Beccuti ${ }^{14}$ Gianfranco Ciardo ${ }^{14}$, Yaqin $\mathrm{Ma}^{14}$, Steve Wanamaker ${ }^{4}$, Timothy J. Close (co-leader) ${ }^{4}$, Nils Stein (leader) ${ }^{6}$

BAC-end sequencing Federica Cattonaro ${ }^{15}$, Vera Vendramin ${ }^{16}$, Simone Scalabrin ${ }^{15}$, Slobodanka Radovic ${ }^{16}$, Rod Wing ${ }^{17}$, Daniela Schulte ${ }^{6}$, Burkhard Steuernagel $^{6}$, Michele Morgante $^{15,16}$, Nils Stein ${ }^{6}$, Robbie Waugh (leader) ${ }^{2}$

Integration of physical/genetic map and sequence resources Thomas Nussbaumer ${ }^{1}$ Heidrun Gundlach ${ }^{1}$, Mihaela Martis ${ }^{1}$, Ruvini Ariyadasa ${ }^{6}$, Naser Poursarebani ${ }^{6}$, Burkhard Steuernage ${ }^{6}$, Uwe Scholz $^{6}$, Roger P. Wise ${ }^{5}$, Jesse Poland ${ }^{18}$, Nils Stein ' ${ }^{6}$, Klaus F. X. Mayer (leader) ${ }^{1}$

Gene annotation Manuel Spannagl ${ }^{1}$, Matthias Pfeifer ${ }^{1}$, Heidrun Gundlach ${ }^{1}$, Klaus F. X. Mayer (leader) ${ }^{1}$

Repetitive DNA analysis Heidrun Gundlach ${ }^{1}$, Cédric Moisy ${ }^{9}$, Jaakko Tanskanen ${ }^{9}$ Simone Scalabrin ${ }^{15}$, Andrea Zuccolo ${ }^{15}$, Vera Vendramin ${ }^{16}$, Michele Morgante ${ }^{15,16}$ Klaus F. X. Mayer (co-leader) ${ }^{1}$, Alan Schulman (leader) ${ }^{9}$

Transcriptome sequencing and analysis Matthias Pfeifer ${ }^{1}$, Manuel Spannagl ${ }^{1}$, Pete Hedley $^{2}$, Jenny Morris ${ }^{2}$, Joanne Russell ${ }^{2}$, Arnis Druka ${ }^{2}$, David Marshall ${ }^{2}$, Micha Bayer ${ }^{2}$, David Swarbreck ${ }^{19}$, Dharanya Sampath ${ }^{19}$, Sarah Ayling ${ }^{19}$, Melanie Febrer ${ }^{19}$, Mario Caccamo $^{19}$, Takashi Matsumoto ${ }^{7}$, Tsuyoshi Tanaka ${ }^{7}$, Kazuhiro Sato ${ }^{8}$, Roger P. Wise ${ }^{5}$, Timothy J. 'Close ${ }^{4}$, Steve Wannamaker ${ }^{4}$, Gary J. Muehlbauer ${ }^{10}$, Nils Stein ${ }^{6}$, Klaus F. X. Mayer (co-leader) ${ }^{1}$, Robbie Waugh (leader) ${ }^{2}$

Re-sequencing and diversity analysis Burkhard Steuernage ${ }^{6}$, Thomas Schmutzer $^{6}$ Martin Mascher ${ }^{6}$, Uwe Scholz ${ }^{6}$, Stefan Taudien ${ }^{13}$, Matthias Platzer ${ }^{13}$, Kazuhiro Sato ${ }^{8}$ David Marshall ${ }^{2}$, Micha Bayer ${ }^{2}$, Robbie Waugh (co-leader) ${ }^{2}$, Nils Stein (leader) ${ }^{6}$

Writing and editing of the manuscript Klaus F. X. Mayer (co-leader) ${ }^{1}$, Robbie Waugh

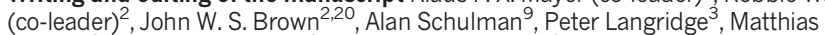
Platzer $^{13}$, Geoffrey B. Fincher ${ }^{21}$, Gary J. Muehlbauer ${ }^{10}$, Kazuhiro Sato ${ }^{8}$, Timothy J. Close $^{4}$, Roger P. Wise ${ }^{5}$ \& Nils Stein (leader) ${ }^{6}$

${ }^{1}$ MIPS/IBIS, Helmholtz Zentrum München, D-85764 Neuherberg, Germany. ${ }^{2}$ The James Hutton Institute, Invergowrie, Dundee DD2 5DE, UK. ${ }^{3}$ Australian Centre for Plant Functional Genomics, University of Adelaide, Glen Osmond 5064, Australia. ${ }^{4}$ Department of Botany \& Plant Sciences, University of California, Riverside, California 92521, USA. ${ }^{5}$ USDA-ARS, Department of Plant Pathology \& Microbiology, lowa State University, Ames, lowa 50011-1020, USA. ' Leibniz Institute of Plant Genetics and Crop Plant Research (IPK), D-06466 Seeland OT Gatersleben, Germany. ${ }^{7}$ National Institute of Agrobiological Sciences, 2-1-2, Kannondai, Tsukuba Ibaraki 305-8602, Japan. ${ }^{8}$ Okayama University, Kurashiki 710-0046, Japan. ${ }^{9}$ MTT Agrifood Research and Institute of Biotechnology, University of Helsinki, FIN-00014 Helsinki, Finland. ${ }^{10}$ University of Minnesota, Department of Agronomy and Plant Genetics, Department of Plant Biology, St Paul, Minnesota 55108, USA. ${ }^{11}$ Institute of Evolution, University of Haifa, Haifa 31905, Israel. ${ }^{12}$ INRA-CNRGV, Auzeville CS 52627, France. ${ }^{13}$ Leibniz Institute of Age Research- Fritz Lipmann Institute (FLI), D-07745 Jena, Germany. ${ }^{14}$ Department of Computer Science \& Engineering, University of California, Riverside, California 92521, USA. ${ }^{15}$ Istituto di Genomica Applicata, Via J. Linussio 51, 33100 Udine, Italy. ${ }^{16}$ Dipartimento di Scienze Agrarie ed Ambientali, Università di Udine, 33100 Udine, Italy. ${ }^{17}$ University of Arizona, Arizona Genomics Institute, Tucson, Arizona 85721, USA. ${ }^{18}$ USDA-ARS Hard Winter Wheat Genetics Research Unit and Kansas State University, Manhattan, Kansas 66506, USA. ${ }^{19}$ The Genome Analysis Centre, Norwich Research Park, Norwich NR4 7UH, UK. ${ }^{20}$ Division of Plant Sciences, University of Dundee at The James Hutton Institute, Invergowrie, Dundee DD2 5DA, UK. ${ }^{21}$ ARC Centre of Excellence in Plant Cell Walls, University of Adelaide, Waite Campus, Glen Osmond, South Australia 5064, Australia. ${ }^{22}$ Department of Computer Science, Corso Svizzera 185, 10149 Torino, Italy. 


\section{METHODS}

Building the physical map. BAC clones of six libraries of cultivar 'Morex ${ }^{9,49}$ were analysed by high information content fingerprinting (HICF) ${ }^{7,9}$. A total of 571,000 edited profiles was assembled using FPC v9.2 ${ }^{8}$ (Supplementary Table 2) (Sulston score threshold of $10^{-90}$, tolerance $=5$, tolerated Q clones $\left.=10 \%\right)$. Nine iterative automated re-assemblies were performed at successively reduced stringency (Sulston score of $10^{-85}$ to $10^{-45}$ ). A final step of manual merging of FPC contigs was performed at lower stringency (Sulston score threshold $10^{-25}$ ) considering genetic anchoring information for markers with a genetic distance $\leq \pm 5 \mathrm{cM}$. This produced 9,265 FPcontigs (approximately 14-fold haploid genome coverage) (Supplementary Table 2).

Genomic sequencing. BAC-end sequencing (BES). BAC insert ends were sequenced using Sanger sequencing (Supplementary Note 2.1). Vector and quality trimming of sequence trace files was conducted using LUCY ${ }^{50}$ (http://www.jcvi.org/ $\mathrm{cms} /$ research/software/). Short reads (that is, $<100 \mathrm{bp}$ ) were removed. Organellar DNA and barley pathogen sequences were filtered by BLASTN comparisons to public sequence databases (http://www.ncbi.nlm.nih.gov/).

BAC shotgun sequencing (BACseq). Seed BACs of the FPC map were sequenced to reveal gene sequence information for physical map anchoring. 4,095 BAC clones were shotgun sequenced in pools of $2 \times 48$ individually barcoded BACs on Roche/ 454 GS FLX or FLX Titanium ${ }^{51,52}$. Sequences were assembled using MIRA v3.2.0 (http://www.chevreux.org/projects_mira.html) at default parameters with features 'accurate', '454', 'genome', 'denovo'. An additional 2,183 gene-bearing BACs (Supplementary Note 3.2) were sequenced using Illumina HiSeq 2000 in 91 combinatorial pools ${ }^{13}$. Deconvoluted reads were assembled using VELVET ${ }^{53}$. Assembly statistics are given in Supplementary Table 4.

Whole-genome shotgun sequencing. Illumina paired-end (PE; fragment size $\sim 350 \mathrm{bp}$ ) and mate-pair (MP; fragment size $\sim 2.5 \mathrm{~kb}$ ) libraries were generated from fragmented genomic DNA ${ }^{54}$ of different barley cultivars ('Morex', 'Barke', 'Bowman', 'Igri') and an S3 single-seed selection of a wild barley accession B1K04-12 $2^{55}$ (Hordeum vulgare ssp. spontaneum). Libraries were sequenced by Illumina GAIIx and Hiseq 2000. Genomic DNA of cultivar 'Haruna Nijo' (size range of 600-1,000 bp) was sequenced using Roche 454 GSFLX Titanium chemistry. Whole-genome shotgun sequence assembly. PE and MP whole-genome shotgun libraries were calibrated for fragment sizes by mapping pairs against the chloroplast sequence of barley (NC_008590) using BWA ${ }^{56}$. Sequences were quality trimmed and de novo assembled using CLC Assembly Cell v3.2.2 (http:// www.clcbio.com/). Independent de novo assemblies were performed from data of cultivars 'Morex', 'Bowman' and 'Barke'.

Transcriptome sequencing. Eight tissues of cultivar 'Morex' (three biological replications each) earmarking stages of the barley life cycle from germinating grain to maturing caryopsis were selected for deep RNA sequencing (RNA-seq). Plant growth, sampling and sequencing is detailed in Supplementary Information (Supplementary Note 6). Further mRNA sequencing data was generated from eight additional spring barley cultivars within a separate study and was used here for sequence diversity analysis (Supplementary Note 8.2).

Genetic framework of the physical map. The genetic framework for anchoring the physical map of barley was built on a single-nucleotide variation (SNV) map ${ }^{57}$ (Supplementary Note 4.3$)$ which provided the highest marker density $(3,973)$ and resolution $(N=360, \mathrm{RIL} / \mathrm{F} 8)$ for a single bi-parental mapping population in barley. Additional high-density genetic marker maps (Supplementary Note 4.3) were compared and aligned on the basis of shared markers. Furthermore, we used genotyping-by-sequencing $(\mathrm{GBS})^{58}$ to generate high-density genetic maps comprising 34,396 SNVs and 21,384 SNVs as well as 241,159 and 184,796 dominant (presence/absence) tags for the two doubled haploid populations Oregon Wolfe Barley $^{14}$ and Morex $\times$ Barke $^{45}$, respectively. Altogether 498,165 marker sequence tags were used (Supplementary Table 11).

Genetic anchoring. Genetic integration of the physical map involved procedures of direct and indirect anchoring.

Direct anchoring. Genetic markers were assigned to BAC clones/BAC contigs by three different procedures (Supplementary Note 4.3 and Supplementary Table 9). 2,032 PCR-based markers from published genetic maps ${ }^{59,60}$ were PCR-screened on custom multidimensional (MD) DNA pools (http://ampliconexpress.com/) obtained from BAC library HVVMRXALLeA ${ }^{9}$. A single haploid genome equivalent of these MD pools was used for multiplexed screening of 42,302 barley ESTderived unigenes represented on a custom $44 \mathrm{~K}$ Agilent microarray as previously described $^{61} .27,231$ barley unigenes, comprising 1,121 with a genetic map position ${ }^{45,62}$, could be assigned to 12,313 BACs. 14,600 clones from BAClibrary HVVMRXALLhA were screened with 3,072 SNP markers on Illumina GoldenGate assays ${ }^{45}$ leading to
1,967 markers directly assigned to $\mathrm{BACs}^{13}$; approximately one third of this information has been included in the present work.

Indirect anchoring. Sequence resources associated with the FPCmap framework provided the basis for extensive in silico integration of genetic marker information (Supplementary Note 4.3 and Supplementary Table 11). Repeat masked BES sequences, sequences of anchored markers and 6,295 sequenced BACs allowed integration of $307 \mathrm{Mb}$ of 'Morex' whole-genome shotgun contigs into the FPC map. Genetic markers and barley gene sequences were positioned to this reference by strict sequence homology association. Overall 8,170 $(\sim 4.6 \mathrm{~Gb})$ $\mathrm{BAC}$ contigs received sequence and/or anchoring information (Supplementary Note 4). 4,556 FPC contigs $(\Sigma=3.9 \mathrm{~Gb})$ were anchored to the genetic framework. Analysis of repetitive DNA and repeat masking. Repeat detection and analysis was undertaken as previously described ${ }^{18,20}$ with the exception of an updated repeat library complemented by de novo detected repetitive elements from barley (Supplementary Note 5).

Gene annotation, functional categorization and differential expression. Publically available barley full-length $\mathrm{cDNAs}^{24}$ and RNA-seq data generated in the project (Supplementary Note 6) were used for structural gene calling (Supplementary Note 7). Full-length cDNAs and RNA-seq data were anchored to repeat masked whole-genome shotgun sequence contigs using GenomeThreader ${ }^{63}$ and CuffLinks $^{64}$, respectively, the latter providing also information of alternatively spliced transcripts. Structural gene calls were combined and the longest ORF for each locus was used as representative for gene family analysis (Supplementary Note 7.1.2)

Gene family clustering was undertaken using OrthoMCL (Supplementary Note 7.1.3) by comparing against the genomes of Oryza sativa (RAP2), Sorghum bicolor, Brachypodium distachyon (v 1.4) and Arabidopsis thaliana (TAIR10 release).

Analysis of differential gene expression (Supplementary Note 7.2) was performed on RNA-seq data using CuffDiff ${ }^{65}$.

Analysis of sequence diversity. Genome-wide SNV was assessed by mapping (BWA v0.5.9-r16 $6^{56}$ ) the original sequence reads of sequenced genotypes to a de novo assembly of cultivar 'Morex'. Sequence reads from RNA-seq were mapped against the 'Morex' assembly. Details are provided in Supplementary Note 8.

49. Yu, Y. et al. A bacterial artificial chromosome library for barley (Hordeum vulgare L.) and the identification of clones containing putative resistance genes. Theor. Appl. Genet. 101, 1093-1099 (2000).

50. Chou, H.-H. \& Holmes, M. H. DNA sequence quality trimming and vector removal. Bioinformatics 17, 1093-1104 (2001).

51. Steuernagel, B. et al. De novo 454 sequencing of barcoded BAC pools for comprehensive gene survey and genome analysis in the complex genome of barley. BMC Genomics 10, 547 (2009).

52. Taudien, S. et al. Sequencing of BAC pools by different next generation sequencing platforms and strategies. BMC Res. Notes 4, 411 (2011).

53. Zerbino, D. R. \& Birney, E. Velvet: algorithms for de novo short read assembly using de Bruijn graphs. Genome Res. 18, 821-829 (2008).

54. Stein, N., Herren, G. \& Keller, B. A new DNA extraction method for high-throughput marker analysis in a large-genome species such as Triticum aestivum. Plant Breed. 120, 354-356 (2001).

55. Hübner, S. et al. Strong correlation of the population structure of wild barley (Hordeum spontaneum) across Israel with temperature and precipitation variation. Mol. Ecol. 18, 1523-1536 (2009).

56. Li, H. \& Durbin, R. Fast and accurate short read alignment with Burrows-Wheeler transform. Bioinformatics 25, 1754-1760 (2009).

57. Comadran, J. et al. A homologue of Antirrhinum CENTRORADIALIS is a component of the quantitative photoperiod and vernalization independent EARLINESS PER SE 2 locus in cultivated barley. Nature Genet. (in the press).

58. Elshire, R. J. et al. A robust, simple genotyping-by-sequencing (GBS) approach for high diversity species. PLoS ONE 6, e19379 (2011).

59. Sato, K., Nankaku, N. \& Takeda, K. A high-density transcript linkage map of barley derived from a single population. Heredity 103, 110-117 (2009).

60. Stein, N. et al. A 1000 loci transcript map of the barley genome - new anchoring points for integrative grass genomics. Theor. Appl. Genet. 114, 823-839 (2007).

61. Liu, H. et al. Highly parallel gene-to-BAC addressing using microarrays. Biotechniques 50, 165-174 (2011).

62. Potokina, E. etal. Gene expression quantitative trait locus analysis of 16,000 barley genes reveals a complex pattern of genome-wide transcriptional regulation. Plant J. 53, 90-101 (2008)

63. Gremme, G., Brendel, V., Sparks, M. E. \& Kurtz, S. Engineering a software tool for gene structure prediction in higher organisms. Inf. Softw. Technol. 47, 965-978 (2005).

64. Trapnell, C et al. Differential gene and transcript expression analysis of RNA-seq experiments with TopHat and Cufflinks. Nature Protocols 7, 562-578 (2012).

65. Trapnell, C. et al. Transcript assembly and quantification by RNA-Seq reveals unannotated transcripts and isoform switching during cell differentiation. Nature Biotechnol. 28, 511-515 (2010). 